\title{
Numeri caratteristici definitivi dei flocculi di calcio per l'Anno Geofisico Internazionale
}

\author{
G. Godotr - F. MazzUCCONI - B. MonstGNorr Fossr
}

\author{
Ricevuto il 15 Gennaio 1966
}

Riassunto. - Si danno i numeri caratteristici definitivi dei flocculi di calcio per l'Anno Geofisico Internazionale, secondo il materiale di osservazione raccolto al World Date Center di Arcetri grazie al programma di collaborazione internazionale.

Questi numeri caratteristici sostituiscono quelli preliminari, già pubblicati in questa sede $\left({ }^{6},{ }^{7}\right)$, i quali vennero stimati in base alle sole ossevazioni eseguite alla torre solare di Arcetri.

Summary. - The definitive character figures of the Ca bright floceuli for the International Geophysical Year are given. The observational material used has been collected at Arcetri World Date Center for the Ca bright flocculi, as a part of the IGY international programme.

These character figures take the place of those already published $\left({ }^{6},{ }^{7}\right)$ according to the only observations carried out at the Arcetri solar tower.

1. - Determinazioni di numeri caratteristici giomalieri dei flocculi solari di calcio sono state pubblicate, per il periodo 1950-1960, in questa sede $\left({ }^{1},{ }^{9}\right)$ e per gli anni 1961, 1963, 1964 nel bollettino ammuale delle osservazioni eseguite all'Osservatorio Astrofisico di Arcetri (10, $\left.{ }^{12}\right)$.

Per queste determinazioni è stato utilizzato il materiale spettroeliografico raccolto alla torre solare di Arcetri nella radiazione della componente $K_{2,3,2}$ della riga $K$ del calcio una volta ionizzato.

Dal 24 Ottobre 1961 al 9 Ottobre 1963, non sono state eseguite determinazioni di numeri caratteristici dei flocculi di calcio dato che le osservazioni alla torre solare sono state sospese per procedere alla sostituzione della cupola e dei servo-meccanismi di comando.

2. - Come è noto, sin dall'inizio dell'Anno Geofisico Internazionale (10 Luglio 1957-31 Dicembre 1958), l'Osservatorio Astrofisico di Arcetri 
è stato Centro Mondiale (World Data Center, WDC) per la raccolta e l'elaborazione dei dati relativi ai floceuli solari in radiazione $\mathbf{K}$ del Ca II.

Durante l'Anno Geofisico Internazionale l'Osservatorio di Arcetri ba ricevuto regolarmente spettroeliogrammi in radiazione $K$ dagli osservatori di Crimea, Kodaikanal, Mc Math Hulbert. Il materiale di osservazione ricevuto da questi tre Osservatori e quello raccolto ad Arcetri copre tutti i giorni del periodo $1^{\circ}$ Luglio 1957-31 Dicembre 1958 con le sole lacune relative ai griorni:

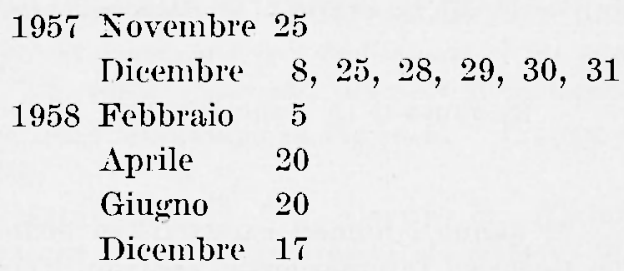

Allo scopo di colmare queste lacune è stata chiesta la collaborazione di tutti gli istituti impegnati in osservazioni sistematiche della cromosfera solare in radiazione $K$ e ciò̀ degrli istituti di Freiburg, Ikomasan, Meudon, Mount Wilson, Tokio, Wendelstein. Con la collaborazione di questi istituti è stato possibile colmare tutte le lacune con la sola eccezione del giorno

1957 Dicembre 8 .

Le caratteristiche del materiale di osservazione ricevuto sono le seguenti:

\begin{tabular}{|c|c|c|}
\hline Istituto & Materiale fotografico & $\begin{array}{l}\text { Diametro } \\
\text { dell'immagine }\end{array}$ \\
\hline Arcetri & originali su lastra & $63 \mathrm{~mm}$ \\
\hline Crimea & 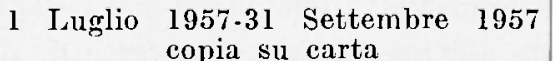 & $43 \mathrm{~mm}$ \\
\hline & $\begin{array}{l}1 \text { Ottobre } \begin{array}{l}1957-31 \text { Dicembre } 1958 \\
\text { copie su film }\end{array}\end{array}$ & $43 \mathrm{~mm}$ \\
\hline I'raunhofer & originali su lastra & $50 \mathrm{~mm}$ \\
\hline Kodaikanal & copie su film & $19 \mathrm{~mm}$ \\
\hline Me Math Hulbert & $\begin{array}{l}\text { 1 Luglio } 1957-31 \text { Dicembre } 1957 \\
\text { copie su film o originali } \\
\text { 1 Gennaio } 1958-31 \text { Dicembre } 1958 \\
\text { originali }\end{array}$ & $\begin{array}{l}17 \mathrm{~mm} \\
17 \mathrm{~mm}\end{array}$ \\
\hline Meudon & copie su lastra & $85 \mathrm{~mm}$ \\
\hline Mount Wilson & copie su lastra & $52 \mathrm{~mm}$ \\
\hline Tokio & copie su carta & $100 \mathrm{~mm}$ \\
\hline Wendelstein & originali su lastra & $45 \mathrm{~mm}$ \\
\hline
\end{tabular}


Le copie e gli originali inviati dai vari istituti sono stati riportati a negativi su lastra e su carta rispettivamente con una o due inversioni fotografiche. Anche gli originali eseguiti ad Arcetri sono stati riportati a negativi su carta con due inversioni fotografiche. Il diametro dell'immagine solare è stato sempre riportato alla misura di $63 \mathrm{~mm}$ per le lastre ed a $120 \mathrm{~mm}$ per le stampe.

Dal materiale di osservazione cosi elaborato, sono state costituite due collezioni (una di lastre ed una di stampe) il più omogenee possibile costituite da uno spettroeliogramma al giorno.

3. - La collezione delle lastre era già stata utilizzata per la compilazione delle mappe dei floceuli solari in radiazione $K$ del Ca II per l'Anno Geofisico Internazionale $\left({ }^{13}, 14,15,{ }^{16}\right)$.

Successivamente abbiamo pensato di utilizzare la collezione delle lastre e quella delle stampe anche per la stima dei numeri caratteristici giornalieri dei floceuli di calcio per l'Anno Geofisico Internazionale in quanto le nuove stime, eseguite su lastre e stampe e su materiale che presenta soltanto una lacuna di un giomo, sarebbero state senz'altro più precise e complete di quelle già pubblicate $\left({ }^{0},{ }^{7}\right)$.

Abbiamo già notato $\left({ }^{7}\right)$ che, sebbene dal $1^{\circ}$ Gennaio 1957 l'attività dei flocculi di calcio venga seguita all'Osservatorio Astrofisico di Arcetri rilevando area e posizione dei singoli fenomeni in quanto questo metodo di determinare l'attività dei flocculi è più informativo della stima del numero caratteristico e sebbene il numero caratteristico sia una funzione nota dell'area proiettata, tuttavia è opportuno continuare a stimare i numeri caratteristici dei floceuli di calcio dato che vi sono elementi per ritenere che, durante fasi peculiari del ciclo solare, $\mathrm{i}$ due diversi metodi diano informazioni di significato diverso $\left({ }^{17}\right)$.

4. - Le stime dei numeri caratteristici sono state sempre eseguite all'Osservatorio Astrofisico di Arcetri attenendosi ai modelli distribuiti dall'Osservatorio di Mount Wilson. I modelli definiscono i punti 0,1 , $2,3,4,5$ della scala dei numeri caratteristici. Ad Arcetri è sempre stato considerato conveniente stimare anche il decimo del numero earatteristico, mentre altri Osservatori preferiscono dare solo i cinque decimi.

I modelli originali di Mount Wilson sono costituiti da positive su carta del diametro di $100 \mathrm{~mm}$. Recentemente ad Arcetri, per migliorare la qualità della stima, si è derivata dai modelli originali di Mount Wilson una serie di modelli costituita da negative su lastra del diametro 
di $63 \mathrm{~mm}$. Tal 1962 i numeri caratteristici sono stati sempre stimati con l'aiuto di questi modelli.

5. - I numeri raratteristici giornalieri dei flocculi di ralcio per l'Anno Geofisico Internazionale sono stati dapprima stimati sulla collezione delle stampe riferendosi ai modelli originali di Mount Wilson. Ia stima sistematica è stata fatta indipendentemente da due osservatori (F. M. e B. M. F.); un terzo osservatore (G. G.) ha saltuariamente controllato le stime. Quando lo searto medio delle singole determinazioni non superava 0.3 unità, veniva fatta una media delle valutazioni dei due osservatori; nei pochi casi in cui veniva riscontrato uno scarto medio di maggior entità, lo spettroeliogramma veniva riesaminato.

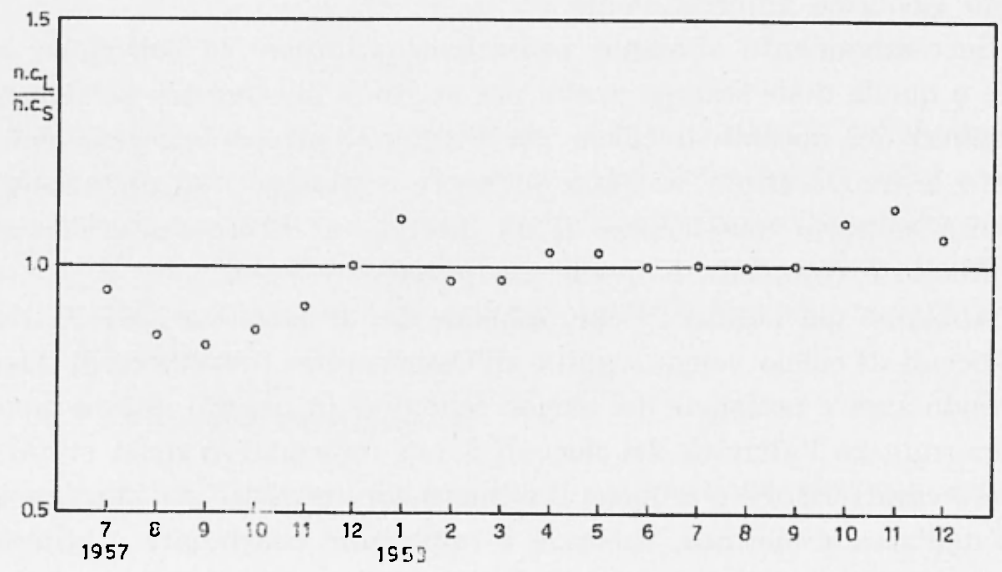

Fị. 1

In un secondo tempo i numeri caratteristici sono stati stimati con lo stesso metodo sulla collezione delle lastre riferendosi ai modelli su lastra.

Dal grafico di Fig. 1 in cui sono riportati i rapporti delle medie mensili dei valori rispettivamente determinati sulle lastre e sulle stampe, si nota che l'accordo fra le due serie di stime è assai soddisfacente.

Per poter confrontare la precisione delle due serie di stime abbiamo applicato il metoro di $\mathrm{A}$. Wald $\left({ }^{18}\right)$.

Indichiamo con $\left(x_{1}, y_{1}\right), \ldots,\left(x_{r}, y_{r}\right), \ldots,\left(x_{n}, y_{n}\right)$ le $n$ coppie (nel nostro caso 548) di determinazioni giornaliere del numero caratteristico eseguite rispettivamente sulle lastre e sulle stampe. Siano

1) $X_{i}$ ed $Y_{i}$ i valori "veri" di $x_{i}$ ed $y_{i}$; 
2) $\bar{x}=\sum x_{i} / n$

3) $\bar{y}=\Sigma y_{i} / n$;

4) $\sigma_{x}^{2}, \sigma_{y}^{2}, \sigma_{x y}$ le varianze delle $x_{i}$, delle $y_{i}$ e le covarianze delle coppie $x_{i}, y_{i}:$

$$
\begin{aligned}
& \sigma_{x}-\sqrt{\frac{1}{n} \sum\left(x_{i}-\bar{x}\right)^{2}} \\
& \sigma_{y}=\sqrt{\frac{1}{n} \sum\left(y_{i}-\bar{y}\right)^{2}} \\
& \sigma_{x y}=\frac{1}{n} \sum\left(x_{i}-x\right)\left(y_{i}-\bar{y}\right) .
\end{aligned}
$$

Definiamo "errori » $\delta_{t}$ e $\Delta_{t}$ le quantità:

$$
\begin{aligned}
& \delta_{i}=x_{i}-X_{i} \\
& \Delta_{i}=y_{i}-Y_{i} .
\end{aligned}
$$

Supponiamo che:

1) gli errori $\delta_{i}$ abbiano distribuzioni identiche e non siano correlati;

2) gli errori $\Delta_{t}$ abbiano distribuzioni identiche e non siano correlati;

3) gli errori $\delta_{i}$ e $\Delta_{i}$ non siano correlati;

4) sia

$$
Y_{i}=\alpha X_{t}+\beta
$$

5) sia

$$
\sum_{1}^{n / 2} x_{i}-\sum_{(n / 2+1)}^{n} x_{i} \mid n>k>0
$$

con $k$ costante.

Posto:

$$
\begin{aligned}
& a_{1}=\left[\sum_{1}^{n / 2} x_{i}-\sum_{(n / 2+1)}^{n} x_{i} \mid / n ;\right. \\
& a_{2}=\left[\sum_{1}^{n / 2} y_{i}-\sum_{(n / 2+1)}^{n} y_{i}{ }_{1} / n ;\right. \\
& a=\frac{a_{2}}{a_{1}} ;
\end{aligned}
$$

indicando con $\sigma_{\searrow}^{2}$ e $\sigma_{\Delta}^{2}$ le varianze dei $\delta_{i}$ e $\Delta_{i}$ si ha, per la legge dei grandi numeri, 


$$
\begin{aligned}
& \sigma_{\partial} \simeq \sqrt{\frac{n}{n-1}\left(\sigma_{x}^{2}-\frac{\sigma_{x y}}{a}\right)}=0.31 . \\
& \sigma_{\Delta} \simeq \sqrt{\frac{n}{n-1}\left(\sigma_{y}^{2}-a \sigma_{x y}\right)}=0.28 .
\end{aligned}
$$

Indicando infine con $r$ il coefficiente di correlazione fra gli $x_{i}$ e gli $y_{i}$ si ha:

$$
r=\frac{\sigma_{x u}}{\sigma_{x} \sigma_{y}}=0.25
$$

Ammettendo verificate le nostre ipotesi, si constata che le due serie di stime hanno praticamente la stessa precisione.

La nuova serie di stime, eseguita sulla collezione completa delle lastre, è stata anche confrontata, sempre applicando il metodo di A. Wald, con la serie di stime già pubblicata $\left({ }^{6},{ }^{7}\right)$, eseguita sulle sole lastre raccolte all'Osservatorio di Arcetri. Naturalmente il confronto è stato possibile soltanto per quei giorni in cui si hanno osservazioni eseguite ad Arcetri.

Indicando ora con $\left(x_{i}, y_{i}\right), \ldots,\left(x_{r}, y_{r}\right), \ldots,\left(x_{n}, y_{n}\right)$ le $n$ coppie (nel nostro caso 245) di determinazioni giornaliere del numero caratteristico eseguite rispettivamente sulla collezione completa delle lastre e sulle sole lastre raccolte all'Osservatorio di Arcetri, si ha

$$
\begin{aligned}
& \sigma_{\partial}=0.4 \\
& \sigma_{\Delta}=0.5 \\
& r=0.26 .
\end{aligned}
$$

Sempre ammettendo che le nostre ipotesi siano verificate, si constata che la precisione della serie di stime eseguita sulla collezione completa delle lastre è maggiore della precisione della serie di stime eseguite sulle sole lastre raccolte all'Osservatorio di Arcetri. Questo fatto era del resto atteso (cfr. n.ro 3): è stata proprio la convinzione che stime eseguite su materiale che presenta soltanto una lacuna di un giomo sarebbero state più precise, oltreché, naturalmente, più complete, che ci ha suggerito di eseguire questa nuova serie di stime.

Notiamo che i bassi coefficienti di correlazione ottenuti nei due confronti sono dovuti al fatto che, nel periodo preso in considerazione, le variazioni reali dei numeri caratteristici sono state dello stesso ordine di grandezza degli scarti medi. 
Tabella 1 - Numeri caratteristici definitivi dei flocculi di calcio per l'Anno Geofisico Internazionale.

(Periodo 1 Luglio 1957-31 Dicembre 1957).

\begin{tabular}{|c|c|c|c|c|c|c|c|c|c|c|c|c|}
\hline \multirow{2}{*}{$\frac{\text { Mese } \rightarrow}{\text { giorno } \downarrow}$} & \multicolumn{2}{|c|}{ Luglio } & \multicolumn{2}{|c|}{ Agosto } & \multicolumn{2}{|c|}{ Settembre } & \multicolumn{2}{|c|}{ Ottobre } & \multicolumn{2}{|c|}{ Novembre } & \multicolumn{2}{|c|}{ Dicembre } \\
\hline & n.c. & 0 & n.c. & 0 & n.c. & o & n.c. & 0 & n.c. & o & n.c. & 0 \\
\hline 1 & 3.7 & A & 3.2 & $A$ & 3.6 & $\mathrm{C}$ & 3.6 & $\mathrm{C}$ & 3.8 & $\mathrm{C}$ & 3.5 & MM \\
\hline 2 & 3.9 & A & 3.2 & $A$ & 3.4 & $M$ & 3.4 & C & 3.8 & $\mathrm{MLI}$ & 3.4 & $A$ \\
\hline 3 & 4.1 & $A$ & 3.2 & $A$ & 3.4 & C & 3.4 & $A$ & 3.7 & 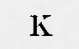 & 3.4 & $A$ \\
\hline 4 & 4.0 & $A$ & 3.4 & MII & 3.4 & $M$ & 3.4 & $A$ & 3.6 & C & 3.4 & $M$ \\
\hline 5 & 4.0 & A & 3.2 & $A$ & 3.6 & $A$ & 3.4 & $A$ & 3.6 & C & 3.4 & $A$ \\
\hline 6 & 3.8 & A & 3.3 & $A$ & 3.6 & A & 3.4 & MM & 3.5 & $\mathrm{C}$ & 3.4 & $\mathrm{~K}$ \\
\hline 7 & 3.8 & $\mathrm{C}$ & 3.2 & $A$ & 3.4 & $A$ & 3.5 & $A$ & 3.4 & $A$ & 3.4 & $\mathbf{K}$ \\
\hline 8 & 3.6 & $A$ & 3.2 & $A$ & 3.3 & $\mathrm{M} M$ & 3.4 & $A$ & 3.4 & $\mathrm{C}$ & - & - \\
\hline 9 & 3.7 & $A$ & 3.4 & $A$ & 3.4 & A & 3.4 & A & 3.4 & $\mathrm{C}$ & 3.2 & $\mathrm{~K}$ \\
\hline 10 & 3.6 & $\mathrm{C}$ & 3.4 & $A$ & 3.4 & $A$ & 3.5 & $A$ & 3.4 & $\mathrm{C}$ & 3.0 & $A$ \\
\hline 11 & 3.5 & $\mathrm{C}$ & 3.2 & $\mathrm{C}$ & 3.4 & $\mathrm{C}$ & 3.6 & $A$ & 3.3 & C & 3.2 & c! \\
\hline 12 & 3.2 & $A$ & 3.4 & A & 3.6 & $\mathrm{C}$ & 3.6 & $A$ & 3.2 & $\mathrm{M} M$ & 3.2 & $\mathrm{~K}$ \\
\hline 13 & 3.2 & A & 3.4 & A & 3.5 & $\mathrm{C}$ & 3.6 & $\kappa$ & 3.4 & $A$ & 3.3 & $\mathrm{~K}$ \\
\hline 14 & 3.2 & $\mathrm{C}$ & 3.6 & C & 3.5 & $\mathrm{C}$ & 3.6 & $A$ & 3.4 & A & 3.2 & $\kappa$ \\
\hline 15 & 3.4 & C & 3.6 & C & 3.5 & C & 3.7 & $A$ & 3.6 & C & 3.2 & K \\
\hline 16 & 3.4 & A & 3.6 & C & 3.4 & A & 3.6 & A & 3.5 & C & 3.2 & A \\
\hline 17 & 3.6 & C & 3.6 & $\mathrm{C}$ & 3.4 & A & 3.6 & A & 3.4 & $\mathbf{K}$ & 3.2 & A \\
\hline 18 & 3.8 & A & 3.7 & МM & 3.4 & A & 3.6 & $\lambda$ & 3.4 & $\mathbf{K}$ & 3.3 & A \\
\hline 19 & 3.8 & MMI & 3.8 & $A$ & 3.5 & A & 3.6 & $\mathrm{C}$ & 3.4 & A & 3.3 & A \\
\hline 20 & 4.2 & C & 3.7 & C & 3.6 & A & 3.6 & C & 3.4 & C & 3.3 & A \\
\hline 21 & 4.0 & C & 3.6 & A & 3.6 & A & 3.6 & $\Lambda$ & 3.4 & C & 3.4 & $\mathrm{C}$ \\
\hline 22 & 4.0 & C & 3.5 & $A$ & 3.6 & $\mathrm{C}$ & 3.6 & C & 3.5 & C & 3.5 & e \\
\hline 23 & 4.2 & $\mathrm{C}$ & 3.4 & MM & 3.8 & $\mathrm{C}$ & 3.6 & A & 3.5 & C & 3.6 & $\mathrm{~K}$ \\
\hline 24 & 4.0 & $A$ & 3.6 & $\mathrm{C}$ & 3.7 & C & 3.6 & $A$ & 3.5 & $\mathbf{K}$ & 3.7 & MLM \\
\hline 25 & 3.8 & A & 3.5 & $\mathrm{C}$ & 3.7 & $\mathrm{C}$ & 3.6 & A & 3.6 & A & 3.7 & MI \\
\hline 26 & 3.7 & A & 3.6 & A & 3.6 & MUI & 3.6 & A & 3.5 & A & 3.8 & $\mathrm{C}$ \\
\hline 27 & 3.7 & A & 3.7 & A & 3.4 & A & 4.0 & C & 3.4 & A & 3.8 & $\mathrm{C}$ \\
\hline 28 & 3.6 & C & 3.6 & A & 3.5 & A & 4.0 & C & 3.4 & $A$ & 3.6 & $M W$ \\
\hline 29 & 3.6 & A & 3.6 & A & 3.6 & $\mathrm{C}$ & 3.7 & $A$ & 3.6 & MIV & 3.6 & $\mathrm{MWV}$ \\
\hline 30 & 3.5 & A & 3.6 & C & 3.6 & $A$ & 3.7 & A & 3.5 & $A$ & 3.6 & $M I V$ \\
\hline 31 & 3.4 & A & 3.6 & A & - & 一 & 3.7 & $A$ & - & $\cdots$ & 3.6 & $M T V$ \\
\hline $\begin{array}{c}\text { Media } \\
\text { mensile }\end{array}$ & \multicolumn{2}{|c|}{3.7} & \multicolumn{2}{|c|}{3.5} & \multicolumn{2}{|c|}{3.5} & \multicolumn{2}{|c|}{3.6} & \multicolumn{2}{|c|}{3.5} & \multicolumn{2}{|c|}{3.4} \\
\hline \multicolumn{13}{|c|}{ Media semestrale } \\
\hline
\end{tabular}




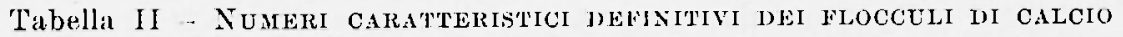
PER L'ANNO GEOHSICO INTERNAZIONALE.

(Periodo 1 Gennaio 1958-30 Giugno 1958).

\begin{tabular}{|c|c|c|c|c|c|c|c|c|c|c|c|c|}
\hline \multirow{2}{*}{$\begin{array}{l}\text { Mese } \rightarrow \\
\text { giorno } \downarrow\end{array}$} & \multicolumn{2}{|c|}{ Gemmaio } & \multicolumn{2}{|c|}{ Febbraio } & \multicolumn{2}{|c|}{ Marzo } & \multicolumn{2}{|c|}{ Aprile } & \multicolumn{2}{|c|}{ Magrgio } & \multicolumn{2}{|c|}{ Giugno } \\
\hline & n.e. & 0 & n.e. & 0 & n.c. & 0 & n.e. & 0 & n.c. & 0 & n.c. & 0 \\
\hline 1 & 3.4 & $\mathrm{~K}$ & 2.9 & $A$ & 3.4 & $\Lambda$ & 3.8 & C & 3.6 & A & 3.7 & $\mathrm{~K}$ \\
\hline 2 & 3.4 & $A$ & 3.0 & K & 3.4 & $K$ & 3.8 & $K$ & 3.6 & $A$ & 3.6 & $K$ \\
\hline 3 & 3.4 & C & 3.0 & $\mathrm{~K}$ & 3.4 & $\Lambda$ & 3.8 & C & 3.8 & $A$ & 3.6 & $A$ \\
\hline+ & 3.4 & $\mathrm{~K}$ & 3.1 & $\mathrm{~K}$ & 3.5 & $\Lambda$ & 3.9 & $\mathbf{K}$ & 3.8 & $\mathrm{C}$ & 3.6 & $A$ \\
\hline 5 & 3.6 & $\mathrm{~K}$ & 3.2 & $\mathrm{~T}$ & 3.6 & $\Lambda$ & 3.8 & C & 3.6 & $A$ & 3.6 & $\mathrm{~K}$ \\
\hline 6 & 3.6 & $\mathrm{c}$ & 3.5 & $\Lambda$ & 3.6 & $\Lambda$ & 3.7 & c & 3.6 & $\mathrm{C}$ & 3.6 & $\mathrm{C}$ \\
\hline 7 & 3.5 & $K$ & 3.6 & $\mathrm{~K}$ & 3.6 & $\mathrm{C}$ & 3.8 & K & 3.6 & MM & 3.7 & $A$ \\
\hline 8 & 3.4 & $A$ & 3.6 & MM & 3.8 & $\mathrm{~K}$ & 3.6 & $\Lambda$ & 3.5 & $A$ & 3.8 & $\mathrm{~K}$ \\
\hline 9 & 3.4 & $A$ & 3.9 & C & 3.8 & C: & 3.7 & $\mathbf{K}$ & 3.6 & $A$ & 3.8 & $A$ \\
\hline 10 & 3.3 & $A$ & 4.0 & c & 3.8 & $\mathrm{~K}$ & 3.6 & $A$ & 3.4 & $A$ & 3.7 & $\mathrm{~K}$ \\
\hline 11 & 3.2 & c & 4.0 & $\mathrm{C}$ & 3.6 & $K$ & 3.6 & $\mathrm{~K}$ & 3.5 & c & 3.6 & C: \\
\hline 12 & 3.4 & $\mathrm{~K}$ & 4.0 & A & 3.6 & $K$ & 3.6 & $\mathrm{~K}$ & 3.4 & $A$ & 3.6 & $\mathrm{~K}$ \\
\hline 13 & 3.7 & $\mathrm{C}$ & 3.8 & A & 3.7 & $\mathbf{K}$ & 3.4 & $\mathrm{C}$ & 3.4 & $A$ & 3.4 & $\mathrm{C}$ \\
\hline $1 t$ & 3.8 & $K$ & 3.6 & $\mathrm{C}$ & 3.6 & $\mathrm{C}$ & 3.4 & $A$ & 3.4 & $A$ & 3.4 & $\mathrm{C}$ \\
\hline 15 & 3.7 & $\mathrm{~K}$ & 3.5 & $\mathrm{~K}$ & 3.6 & $K$ & 3.2 & C & 3. 2 & $\mathrm{~K}$ & 3.4 & $\mathrm{C}$ \\
\hline 16 & 3.6 & $A$ & 3.4 & $\mathrm{~K}$ & 3.6 & $\mathrm{~K}$ & 3.2 & $\mathrm{~K}$ & 3.3 & C & 3.4 & $A$ \\
\hline 17 & 3.4 & $\Lambda$ & 3.4 & $\mathrm{~K}$ & 3.4 & $K$ & 3.4 & K & 3.4 & C & 3.2 & $M M$ \\
\hline 18 & 3.4 & $\Lambda$ & 3.4 & $\mathrm{~K}$ & 3.4 & $\mathrm{C}$ & 3.4 & $\mathrm{~K}$ & 3.4 & $\mathrm{C}$ & 3.0 & $\mathrm{C}$ \\
\hline 19 & 3.6 & $\mathrm{C}$ & 3.4 & $K$ & 3.4 & $K$ & 3.4 & $\Lambda$ & 3.2 & A & 3.1 & $\mathrm{~K}$ \\
\hline 20 & 3.5 & $\mathrm{C}$ & 3.4 & $A$ & 3.4 & $\mathrm{~K}$ & 3.6 & $M W$ & 3.2 & $A$ & 3.2 & $\mathrm{M} W$ \\
\hline 21 & 3.4 & C & 3.4 & $\mathrm{~K}$ & 3.4 & $\mathrm{~K}$ & 3.5 & $A$ & 3.2 & $A$ & 3.2 & $\mathrm{C}$ \\
\hline 22 & 3.4 & $\Lambda$ & 3.3 & $K$ & 3.2 & $A$ & 3.6 & 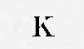 & 3.2 & $\mathrm{C}$ & 3.2 & $\mathrm{C}$ \\
\hline 23 & 3.3 & $\Lambda$ & 3.2 & $\mathrm{~K}$ & 3.3 & $\mathrm{~K}$ & 3.6 & $\mathrm{C}$ & 3.2 & $\mathrm{~K}$ & 3.3 & $\mathrm{C}$ \\
\hline 24 & 3.2 & $A$ & 3.4 & $\mathrm{~K}$ & 3.4 & $\mathrm{C}$ & 3.6 & $\Lambda$ & 3.3 & $A$ & 3.4 & $\mathrm{C}$ \\
\hline 25 & 3.2 & $A$ & 3.4 & $\mathrm{~K}$ & 3.4 & c & 3.6 & $\Lambda$ & 3.3 & $\mathrm{~K}$ & 3.5 & $A$ \\
\hline 26 & 3.2 & $\mathrm{~K}$ & 3.4 & $\mathrm{c}$ & 3.4 & (: & 3.6 & $\Lambda$ & 3.5 & $A$ & 3.6 & $\mathrm{~K}$ \\
\hline 27 & 3.2 & $A$ & 3.4 & $A$ & 3.6 & $\mathrm{~K}$ & 3.7 & C & 3.5 & $A$ & 3.8 & $\mathrm{C}$ \\
\hline 28 & 3.2 & $A$ & 3.2 & $\mathrm{C}$ & 3.7 & $\mathrm{~K}$ & 3.6 & $\Lambda$ & 3.6 & $A$ & 3.8 & C \\
\hline 29 & 3.0 & $A$ & - & $\ldots$ & 3.6 & $\mathrm{~K}$ & 3.6 & $A$ & 3.6 & $A$ & 4.0 & $\mathrm{C}$ \\
\hline 30 & 3.0 & $A$ & - & $\ldots$ & 3.6 & $C^{\prime}$ & 3.6 & A & 3.6 & $\mathrm{~K}$ & 3.7 & $\mathrm{C}$ \\
\hline 31 & 3.0 & $\mathrm{~K}$ & --- & -- & 3.7 & $\mathrm{~K}$ & -- & - & 3.6 & $A$ & $\ldots$ & - \\
\hline $\begin{array}{c}\text { Media } \\
\text { mensile }\end{array}$ & \multicolumn{2}{|c|}{3.4} & \multicolumn{2}{|c|}{3.4} & \multicolumn{2}{|c|}{3.5} & \multicolumn{2}{|c|}{3.6} & \multicolumn{2}{|c|}{3.4} & \multicolumn{2}{|c|}{3.5} \\
\hline
\end{tabular}


'Tabella III - NuMERI CARATTERISTICI DEFINITIVI DEI Floccei DI CALCIO PER L'ANNO GEORLICO INTERNAZIONALE.

(Periodo I lagrlio 1958-31 10icembre 1958).

\begin{tabular}{|c|c|c|c|c|c|c|c|c|c|c|c|c|}
\hline \multirow{2}{*}{$\frac{\text { Mese } \rightarrow}{\text { giorno } \downarrow}$} & \multicolumn{2}{|c|}{ Luglio } & \multicolumn{2}{|c|}{ Agosto } & \multicolumn{2}{|c|}{ Settembre } & \multicolumn{2}{|c|}{ Ottobre } & \multicolumn{2}{|c|}{ Novembre } & \multicolumn{2}{|c|}{ Dicembre } \\
\hline & n.c. & 0 & n.e. & 0 & in.c. & 0 & n.c. & 0 & n.c. & 0 & nic. & 0 \\
\hline 1 & 3.5 & C & 3.8 & $A$ & 3.8 & IIII & 3.4 & c & 3.4 & c & 3.7 & K \\
\hline 2 & 3.6 & $A$ & 3.8 & $A$ & 3.6 & A & 3.6 & c & 3.5 & C & 3.6 & $\mathbf{K}$ \\
\hline 3 & 3.6 & $\Lambda$ & 3.7 & IIMI & 3.6 & A & 3.5 & A & 3.6 & (! & 3.6 & C \\
\hline 4 & 3.7 & $\Lambda$ & 3.6 & $A$ & 3.6 & $A$ & 3.6 & $\Lambda$ & 3.6 & ( & 3.6 & $A$ \\
\hline 5 & 3.7 & $\mathrm{~K}$ & 3.6 & $A$ & 3.6 & $\Lambda$ & 3.6 & $\mathrm{C}$ & 3.4 & A & 3.6 & $A$ \\
\hline 6 & 3.8 & MII & 3.6 & $A$ & 3.6 & $\Lambda$ & 3.6 & $\mathrm{c}$ & 3.5 & K & 3.6 & $A$ \\
\hline 7 & 3.8 & $\lambda$ & 3.6 & MII & 3.6 & $\mathrm{C}$ & 3.6 & MIII & 3.4 & $\mathrm{~K}$ & 3.6 & K \\
\hline 8 & 3.8 & $\Lambda$ & 3.6 & A & 3.4 & C & 3.5 & A & 3.4 & $A$ & 3.6 & C \\
\hline 9 & 3.8 & $A$ & 3.6 & A & 3.5 & $\Lambda$ & 3.4 & $A$ & 3.2 & $\mathrm{C}$ & 3.6 & MM \\
\hline 10 & 3.6 & $A$ & 3.5 & MII & 3.6 & $\mathrm{C}$ & 3.6 & $\mathrm{C}$ & 3.1 & (: & 3.5 & C \\
\hline 11 & 3.6 & $A$ & 3.6 & $\Lambda$ & 3.6 & $A$ & 3.6 & A & 3.0 & (: & 3.6 & $\mathrm{~K}$ \\
\hline 12 & 3.6 & $A$ & 3.6 & $\Lambda$ & 3.8 & C & 3.6 & C & 3.2 & $c$ & 3.6 & $A$ \\
\hline 13 & 3.4 & $\mathrm{C}$ & 3.5 & $\mathrm{C}$ & 3.8 & $A$ & 3.7 & C & 3.4 & C & 3.6 & $\mathrm{MM}$ \\
\hline 14 & 3.3 & A & 3.6 & $A$ & 3.8 & $\mathrm{O}$ & 3.8 & $\Lambda$ & 3.4 & IIMI & 3.5 & $M$ \\
\hline 15 & 3.4 & $A$ & 3.5 & MII & 3.8 & A & 3.8 & $A$ & 3.4 & $A$ & 3.5 & $M \|$ \\
\hline 16 & $3 . \overline{5}$ & $\Lambda$ & 3.5 & $A$ & 3.8 & $\Lambda$ & 3.8 & IIII & 3.5 & $K$ & 3.4 & K \\
\hline 17 & 3.6 & $\mathrm{C}$ & 3.5 & $M$ & 3.8 & c & 3.8 & A & 3.4 & $A$ & 3.4 & $\mathrm{IIW}$ \\
\hline 18 & 3.6 & $A$ & 3.6 & $\Lambda$ & 3.7 & $A$ & 3.8 & $A$ & 3.6 & $\Lambda$ & 3.2 & C \\
\hline 19 & 3.6 & $A$ & 3.6 & $A$ & 3.6 & $\Lambda$ & 3.8 & C & 3.6 & $A$ & 3.3 & $K$ \\
\hline 20 & 3.6 & C & 3.7 & $A$ & 3.6 & $\Lambda$ & 3.8 & C & 3.6 & $A$ & 3.2 & $\mathrm{C}$ \\
\hline 21 & 3.7 & A & 3.7 & $\mathrm{C}$ & 3.6 & c & 3.8 & $A$ & 3.6 & $C^{\prime}$ & 3.2 & $\mathrm{C}$ \\
\hline 22 & 3.7 & $\mathrm{C}$ & 3.7 & C & 3.4 & $A$ & 3.6 & $\Lambda$ & 3.6 & (! & 3.2 & $\mathrm{~K}$ \\
\hline 23 & 3.6 & () & 3.8 & $\Lambda$ & 3.4 & $\lambda$ & 3.5 & C & 3.4 & $\mathrm{~K}$ & 3.3 & C \\
\hline 24 & 3.6 & C & 3.8 & $\mathrm{~K}$ & 3.4 & $\lambda$ & 3.4 & A & 3.4 & $\mathrm{~K}$ & 3.4 & $\mathrm{~K}$ \\
\hline 25 & 3.6 & $A$ & 3.8 & $\Lambda$ & 3.4 & $\Lambda$ & 3.4 & $\Lambda$ & 3.4 & K & 3.5 & $\mathrm{C}$ \\
\hline 26 & 3.8 & $A$ & 3.9 & $\Lambda$ & 3.2 & $\Lambda$ & 3.2 & $\mathrm{~K}$ & 3.5 & $\mathrm{~K}$ & 3.6 & $\mathrm{~K}$ \\
\hline 27 & 3.8 & C & 4.0 & $\Lambda$ & 3.3 & c & 3.2 & A & 3.4 & $\mathrm{~K}$ & 3.6 & $\mathrm{~K}$ \\
\hline 28 & 3.8 & $\Lambda$ & 3.8 & A & 3.3 & C & 3.3 & A & 3.4 & $\mathrm{~K}$ & 3.8 & K \\
\hline 29 & 3.8 & C & 3.8 & $A$ & 3.3 & $A$ & 3.4 & $A$ & 3.6 & $A$ & 3.8 & $\kappa$ \\
\hline 30 & 3.8 & $A$ & 3.8 & IIII & 3.4 & $A$ & 3.4 & A & 3.6 & $\mathrm{C}$ & 3.7 & $\mathrm{~K}$ \\
\hline 31 & 3.8 & $A$ & 3.6 & C & - & $\cdots$ & 3.4 & $\mathrm{C}$ & -- & - & 3.6 & $\mathrm{~K}$ \\
\hline $\begin{array}{c}\text { Media } \\
\text { mensile }\end{array}$ & \multicolumn{2}{|c|}{3.6} & \multicolumn{2}{|c|}{3.7} & \multicolumn{2}{|c|}{3.6} & \multicolumn{2}{|c|}{3.6} & \multicolumn{2}{|c|}{3.4} & \multicolumn{2}{|c|}{3.5} \\
\hline
\end{tabular}


In seguito ai confronti che abbiamo eseguito, abbiamo deciso di prendere come numeri caratteristici definitivi per l'Anno Geofisico Intermazionale le medie fra la nuova serie di determinazioni sulle lastre e quella sulle stampe. Queste medie sono riportate nelle Tabelle I-III.

Nelle Tabelle è indicata anche la fonte dello spettroeliogramma analizzato secondo il codice:
A Arcetri
C Crimea
K Kodaikanal
MIM Me Math Hulbert
M Meudon
MW Mt. Wilson
T Tokio.

Notiamo che lo scarto medio fra la nuova stima e quella già pubblicata rientra generalmente nel limite accettato non superando il valore 0.3. Nella seguente Tabella sono riportati i giorni per cui lo scarto medio supera il valore 0.3 .

Tabella IV

\begin{tabular}{|c|c|}
\hline Scarto medio & Giorni \\
\hline $0.3<$ s.m. $\leqslant 0.4$ & 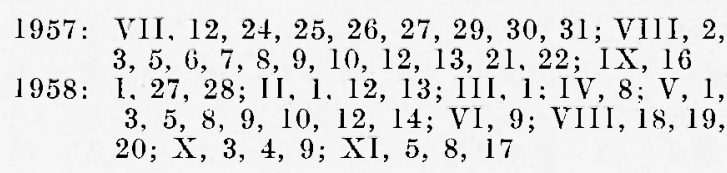 \\
\hline $0.4<\mathrm{s.m} . \leqslant 0.5$ & $\begin{array}{ll}1957: & \text { VII, 27, 29; VIII, } 10,22 \\
1958: & \text { II, 20;III, 4, 22; IV, 19, 21, 24;V, I9, } \\
& 21 ; \text { VI, } 16 ; X, 8,14,15,17,18,22,24, \\
& 29 ; \text { XI, } 15\end{array}$ \\
\hline $0.5<\mathrm{s} . \mathrm{m} . \leqslant 0.6$ & $\begin{aligned} 1957: & \text { VIII, } 21 \\
1958: & \text { III, 3, 5, 6; IV, } 14 ; \mathrm{V}, 20 ; \mathrm{X}, 11,21,25, \\
& 27,28,30\end{aligned}$ \\
\hline $0.6<$ s.m. $\leqslant 0.7$ & 1958: II, 27. \\
\hline
\end{tabular}




\section{BIBIJOGRAFIA}

(1) Godor. G., Numeri caratteristici dei focculi didrogeno e di calcio e dei filamenti d'idrogeno per gli anni 1950-51-52, "Ann. Geof. ", 6. 199, (1953).

(2) Idem per l'anno 1953, l. c., 7, 135 (1954).

(3) Idem per l'anno 1954, l. c., 8, 325, (1955).

(4) Idem per l'anno 1955, 1. c., 9, 299, (1956).

(5) Idem per l'anno 1956, l. c., 10, 133, (1957) (In collaborazione con M. P. Strocchi).

(6) Idem per lanno 1957, l. c., 10, 235, (1957).

(7) Idem per l'anno 1958, 1. c., 12, 197, (1959).

$\left.{ }^{8}\right)$ Idem per l'anno 1959, l. c., 13. 231, (1960).

$\left.{ }^{9}\right)$ Idem per l'anno 1960, 1. c., 14, 319, (1961).

${ }^{(10)}$ Osservazioni eseguite all Osservatorio Astrofisico di Arcetri durante lanno 1961, "Oss. e Mem. dell'Oss. Astrof. di Arcetri», 77, (1962).

(11) Idem per lanno 1963, I. c., 79, (1964).

(12) Idem per l'anno 1964, l. c., 80, (1965).

(13) Godoli, G., Mappe dei flocculi solari in radiazione $K$ del Ca $I I$ per linno Geofisico Internazionale. 1: Semestre 1 Iuglio 195r-31 Dicembre 195\%, "Oss. e Mlen. dell'Oss. Astrof. di Arcetri", 73. (1961).

(14) Idem per il Semestre 1 Gennaio 1958-30 Giugno 1958, l. c., 75. (1961).

(15) Idem per il Semestre l I.uglio 1958-31 Dicembre 1958, 1. c., 76, (1962).

${ }^{16}$ ) IGY solar activity maps $D_{1}$, "Annals of the International Geoplyssical Year ", 21, Pergamon Press, Oxford, (1961).

(17) Godoli G., Attirità dei focculi didrogeno e di calcio e dei filamenti di idrogeno per il periodo 1 Luglio 195\%-30 Giugno 1958, "Boll. di Geod. e Scienze Affini », 17, 487, (1958).

(18) WALD A., The fitting of straight lines if both rariables are subject to errors, "Ann. Math. Statistics", 11. 284, (1940). 